
\title{
Reseacch S Suare \\ Reduced risk of serious pneumococcal infection up to 10 years after 7 -valent pneumococcal conjugate vaccine in arthritis patients
}

Johanna Nagel ( $\sim$ johanna.nagel@med.lu.se )

Lund University, Skåne University Hospital

G Jönsson

Lund University, Skåne University Hospital

J-Å Nilsson

Lund University, Skåne University Hospital

C Manuswin

Skåne University Hospital

$M$ Englund

Lund University

T Saxne

Lund University, Skåne University Hospital

MC Kapetanovic

Lund University, Skåne University Hospital

\section{Research Article}

Keywords: pneumococcal infection, conjugated pneumococcal vaccine, vaccine efficacy, inflammatory arthritis

Posted Date: February 21st, 2022

DOI: https://doi.org/10.21203/rs.3.rs-1145857/v2

License: (a) (1) This work is licensed under a Creative Commons Attribution 4.0 International License. Read Full License 


\section{Abstract}

Background: To examine rates of serious pneumococcal infections up to 10 years after vaccination with 7-valent conjugated pneumococcal vaccine (PCV7) in patients with arthritis compared to non-vaccinated arthritis patients.

Methods: In total, 595 adult arthritis patients (rheumatoid arthritis; RA=342, 80\% women and spondylarthropathy; SpA=253, 45\% women) received one dose of PCV7. Mean age/disease duration were $62 / 16$ and 51/14 years, respectively. For each patient, 4 matched reference subjects were identified.

At vaccination, 420 patients received bDMARDs (anti-TNF=330, tocilizumab=15, abatacept $=18$, anakinra $=1$, rituximab $=56)$. Methotrexate was given as monotherapy $(n=86)$ or in combination with bDMARD ( $n=220) .89$ SpA patients received NSAIDs without DMARD.

The Skåne Healthcare Register was searched for ICD-10 diagnostic codes for pneumococcal infections (pneumonia, lower respiratory tract infection, septicemia, meningitis, septic arthritis) between January 2000 and December 2018. Frequency of infections after vs before vaccination were calculated (relative risks). Relative risk ratio (RRR) and relative risk reduction (1-RRR) were calculated comparing patients vs non-vaccinated references. Kaplan-Meier and Cox regression were used to investigate time to first event and predictors of infections.

Results: Among vaccinated RA and SpA patients, there was a significant relative risk reduction of pneumonia and all serious infections; $53 \%$ and $46 \%$, respectively. There was no significant difference in time to first pneumonia or all serious infections after vaccination between patients and references. Higher age, RA diagnosis and concomitant prednisolone were associated with infections.

Conclusion: One dose of pneumococcal conjugate vaccine may decrease risk of serious pneumococcal infection up to 10 years in patients with arthritis receiving immunomodulating treatment.

Clinical trial registration number: EudraCT EU 2007-006539-29 and NCT 00828997

Date of study registration: $26 / 01 / 2009$

Date of first patient inclusion: 08/05/2008

\section{Introduction}

Long-term protective effect following pneumococcal immunization is likely due to a combination of protective serum antibody levels, immunological memory and herd immunity. (1) The vast majority of studies evaluating pneumococcal vaccine efficacy in patients with inflammatory rheumatic disease (IRD) with immunosuppressive treatments are assessing serotype specific antibody levels and antibody functionality; opsonophagocytic activity (OPA) as surrogate measures of protective effect. (2) However, the correlation between postvaccination IgG serum antibody levels, antibody function and prediction of 
long term protection against infection remain debatable. (3) The 23-valent polysaccharide vaccine (PPV23) and the conjugated 7- or 13-valent vaccines (PCV) are alike included in the present recommendations for patients with IRD. (4) Both types of vaccine have been reported to have satisfying short-term immunogenicity and safety, but with impaired antibody response among patients with certain ongoing disease modifying anti rheumatic treatments (DMARDs) such as methotrexate and rituximab. (5) The PPV23 is a polysaccharide vaccine inducing a T-cell independent antibody response, theoretically without development of an immunological memory. The conjugated PCV vaccine induces a T-cell dependent antibody response, thus including the forming of antigen specific memory B cells (MBCs). (6) Measurement of these MBCs have been performed in studies of different patient populations receiving PCV, but the predictive value of MBCs for the persistence of humoral immunity has not yet been established or standardized. (3) Studies investigating clinical efficacy of pneumococcal vaccination among patients with IRD are scarce. (7) In the present study we present one of few evaluations of putative serious pneumococcal infection rates up to 10 years before and after administration of heptavalent conjugated pneumococcal vaccine (PCV7) in patients with inflammatory arthritis, compared to non-vaccinated arthritis patients.

\section{Methods}

Adult patients with rheumatoid arthritis (RA) and spondylarthropathy ( $\mathrm{SpA}$ ) including psoriatic arthritis (PsA) patients, at the Department of Rheumatology at Skåne University Hospital, Sweden with established diagnoses and regular follow-up were eligible for the study. The inclusion criteria partly consisted of 6 pre-set treatment groups. RA patients were to have ongoing treatment with 1) methotrexate (MTX), 2) MTX and bDMARD, or 3) bDMARD as monotherapy. SpA patients were to have ongoing treatment with 1) bDMARD, 2) MTX and bDMARD, or 3) NSAID only. All patients, apart from the NSAID treated SpA patients, could also have other concurrent ongoing sDMARD treatment and or glucocorticoids. Patients coming for their regular check-up and meeting the inclusion criteria were consecutively offered to enter the study. Between May 2008 and February 2012, 609 patients received one single intramuscular dose of PCV7. 595 patients were included in the present investigation, 13 patients moved from the area. Written informed constent was obtained from all patients before inclusion in the study. Ethical approval for the study was received from the regional ethics committee in Lund, Sweden. All methods were performed in accordance with the relevant guidelines and regulations.

For each vaccinated patient, 4 reference subjects were identified in the Skåne Health Care Register including all ICD-codes from the entire health care system. The reference subjects $(n=2379)$ were matched for arthritis diagnosis, age, sex, and residency in the same geographical area at least 3 years before and after the index date; that is the date of vaccination of their corresponding patient. References were presumed to be non-vaccinated for pneumococci with a conjugated pneumococcal vaccine, following the current national guidelines. The reference subjects were unaware of their participation and the ethical permission did not include review of their medical records. 
The Skåne Healthcare Register was searched for ICD-10 diagnostic codes for serious putative pneumococcal infections between January 2000 and December 2018 for both patients and reference subjects. The outcome events included ICD-codes corresponding to community acquired pneumonia (J13.9, J18.0, J18.1, J18.9), lower respiratory tract infection (J22.9), septicemia (A40.3), meningitis (G00.1), septic arthritis (M00.1, M00.2B/2C/2D/2F/2G/2H/2X), and unspecified disease caused by Spneumoniae (B95.3)

Pre-vaccination infections were defined as infections occurring from 31 December 2004 until 1 month after date of vaccination, with regard to expected time to protective effect. (8) Infections occurring one month after vaccination until 31st of December 2018 were defined as post vaccination infections.

ICD-codes corresponding to pneumonia $(\mathrm{J} 13, \mathrm{~J} 18)$ were calculated separately as well as together with ICD-codes corresponding to other serious pneumococcal infections. Relative risk (RR) of these two endpoints were calculated by comparing event rates after vs before vaccination. The ratio of relative risk (RRR) was calculated comparing the RR of vaccinated patients vs the non-vaccinated reference subjects. Relative risk reduction corresponds to 1-RRR. A generalized estimated equation (GEE) was used to handle correlated data for several events in the same individual and included time of follow up as a covariate. Absolute risk reduction (ARR) and number needed to treat (NNT), were calculated when applicable. Survival analysis (Kaplan-Meier) and Cox regression analysis were performed to investigate time to first event before and after vaccination, time to death and predicting factors of contracting infection after vaccination.

\section{Results}

342 RA patients and 253 SpA patients were included. Mean age was 62 and 51 years, $80 \%$ vs $45 \%$ were women and mean disease duration was 16 and 14 years, respectively.

At the time of vaccination, 420 patients were treated with bDMARDs including anti-TNF agents $(n=330)$, tocilizumab $(n=15)$, abatacept $(n=18)$, anakinra $(n=1)$ and rituximab $(n=56)$. Methotrexate (MTX) was given as monotherapy $(n=86)$, or in combination with bDMARD $(n=220)$. Eighty-nine of the SpA patients were treated with NSAIDs without DMARD. Thirty percent $(n=176)$ of the patients were treated with prednisolone, mean weekly dose 41 (1-140) mg. For more demographics, see Table 1. 
Table 1

Demographics and disease characteristics at vaccination

\begin{tabular}{|c|c|c|c|}
\hline & $\begin{array}{l}\text { All vaccinated } \\
\text { patients }\end{array}$ & RA & SpA \\
\hline $\mathrm{n}$ & 595 & 342 & 253 \\
\hline Age, years & $\begin{array}{l}57.0 \text { (13.1) 22.5- } \\
87.3\end{array}$ & $\begin{array}{l}61.5(12.2) \text { 24.7- } \\
87.3\end{array}$ & $\begin{array}{l}51.0(11.8) 22.5- \\
76.6)\end{array}$ \\
\hline Gender, \% female & 64.7 & 79.5 & 44.7 \\
\hline Smoking \% & 17.5 & 18.5 & 16.2 \\
\hline Disease duration, years & $15.3(11.6) 0-57$ & $16.3(11.9) 0-57$ & $14.0(11.1) 0-53$ \\
\hline DAS28 (SD) range & 3.3 (1.3) 0-7.5 & $3.7(1.2) 0.6-7.5$ & 2.8 (1.2) 0-6.4 \\
\hline DAS28CRP (SD) range & $2.9(1.1) 1.0-7.0$ & $3.2(1.1) 1.0-7.0$ & $2.6(1.0) 1.0-5.9$ \\
\hline HAQ (SD) range & $0.7(0.6) 0-2.6$ & $0.9(0.7) 0-2.6$ & $0.5(0.5) 0-2.1$ \\
\hline RF \% & - & 82.2 & - \\
\hline Anti-CCP positive $\%$ & - & 78.9 & - \\
\hline HLA-B27\% & - & - & 48.2 \\
\hline MTX \% & 51.6 & 65.8 & 32.0 \\
\hline bDMARD \% & 70.6 & 74.9 & 64.8 \\
\hline MTX+bDMARD \% & 37.0 & 40.6 & 32.0 \\
\hline bDMARD monoterapi \% & 33.6 & 34.2 & 32.8 \\
\hline Rituximab \% & 9.4 & 16.4 & 0 \\
\hline Rituximab and MTX \% & 4.5 & 7.9 & 0 \\
\hline NSAID without DMARD & 15 & - & 35.2 \\
\hline $\begin{array}{l}\text { Prednisolone use at } \\
\text { vaccination \% }\end{array}$ & 29.6 & 41.5 & 13.4 \\
\hline Prednisolone mg/week & 40.7 (21.7) 1-140 & 41.9 (22.5) 1-140 & 35.9 (17.3) 10-87.5 \\
\hline \multicolumn{4}{|c|}{$\begin{array}{l}\text { Values given as percentage or mean (SD), range. All treatment counts were ongoing at vaccination. } \\
\text { RA, Rheumatoid Arthritis; SpA, spondylarthropathy; DAS28, Disease Activity Score using } 28 \text { joint } \\
\text { counts; CRP, C-reactive protein; HAQ, Health Assessment Questionnaire; RF, rheumatoid factor; anti- } \\
\text { CCP, anti-cyclic citrullinated peptide; HLA, human leucocyte antigen; MTX, methotrexate; bDMARD, } \\
\text { biologic disease modifying anti rheumatic drug, including anti-TNF, abatacept, tocilicumab, rituximab, } \\
\text { and one patient on anakinra; NSAID, non-steroidal antirheumatic drug. }\end{array}$} \\
\hline
\end{tabular}

The number of events, relative risk (RR), ratio of relative risk (RRR), relative risk reduction (1-RRR), absolute risk reduction (ARR) and number needed to treat/vaccinate (NNT) in order to prevent one event, 
are all summarized in Table 2. 
Table 2

Number of events, relative risk (RR), ratio and reduction of relative risk (RRR)/(1-RRR).

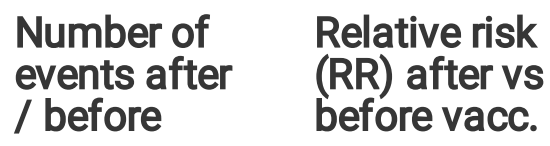

vacc. (95\% Cl), GEE

\section{Ratio of relative risk (RRR)}

vaccinated patients vs reference subjects, (1-RRR)

\section{PNEUMONIA}

$\begin{array}{llllll}\begin{array}{l}\text { All patients } \\ \text { vaccinated } \\ \text { (n=595) }\end{array} & 76 / 42 & 1.74(1.14-2.64) & 0.47(0.28-0.77) & 53 \%^{*} & - \\ \begin{array}{l}\text { All references } \\ \text { subjects } \\ (n=2379)\end{array} & 263 / 77 & \begin{array}{l}3.65(2.70- \\ 4.93)\end{array} & & \\ \begin{array}{l}\text { RA patients } \\ \text { vaccinated } \\ \text { (n=342) }\end{array} & 66 / 33 & 1.83(1.15-2.91) & 0.54(0.31-0.93) & 46 \%^{*} & - \\ \begin{array}{l}\text { RA reference } \\ \text { subjects } \\ (n=1376)\end{array} & 202 / 59 & 3.43(2.51-4.68) & & \\ \begin{array}{l}\text { SpA patients } \\ \text { vaccinated } \\ (n=253)\end{array} & 10 / 9 & 1.01(0.34-3.41) & 0.30(0.09-1.04) & 70 \% & 2 \%(50) \\ \begin{array}{l}\text { SpA } \\ \text { reference } \\ \text { subjects }(n \\ =1003)\end{array} & 61 / 18 & 7.56(3.60-5.88) & & \\ \end{array}$

\section{ALL SERIOUS PNEUMOCOCCAL INFECTIONS}

$\begin{array}{lcccc}\begin{array}{l}\text { All patients } \\ \text { vaccinated } \\ (n=595)\end{array} & 85 / 48 & 1.72(1.12-2.63) & 0.54(0.33-0.87) & 46 \% * \\ \begin{array}{l}\text { All reference } \\ \text { subjects } \\ (n=2379)\end{array} & 307 / 103 & 3.09(2.33-4.10) & & \\ \begin{array}{l}\text { RA patients } \\ \text { vaccinated } \\ (n=342)\end{array} & 74 / 39 & 1.77(1.11-2.82) & 0.63(0.37-1.08) & 37 \% \\ & & & & \end{array}$

RA reference $\quad 232 / 81 \quad 2.84(2.13-3.78)$

subjects

$(n=1376)$

SpA patients $\quad 11 / 9$

vaccinated

$1.18(0.38-$

3.73)

$0.33(0.10-1.13)$

$67 \%$

$3 \%,(34)$

$(n=253)$ 


\begin{tabular}{|c|c|c|c|c|c|}
\hline & $\begin{array}{l}\text { Number of } \\
\text { events after } \\
\text { / before } \\
\text { vacc. }\end{array}$ & $\begin{array}{l}\text { Relative risk } \\
\text { (RR) after vs } \\
\text { before vacc. } \\
\text { (95\% Cl), GEE }\end{array}$ & $\begin{array}{l}\text { Ratio of relative risk } \\
\text { (RRR) } \\
\text { vaccinated patients } \\
\text { vs reference subjects, } \\
(95 \% \mathrm{Cl})\end{array}$ & $\begin{array}{l}\text { Relative } \\
\text { risk } \\
\text { reduction } \\
\text { (1-RRR) }\end{array}$ & $\begin{array}{l}\text { Absolute } \\
\text { risk } \\
\text { reduction, } \\
\text { ARR } \\
\text { (NNT) }\end{array}$ \\
\hline $\begin{array}{l}\text { SpA } \\
\text { reference } \\
\text { subjects } \\
(n=1003)\end{array}$ & $75 / 22$ & $\begin{array}{l}7.19(3.29- \\
15.73)\end{array}$ & & & \\
\hline \multicolumn{6}{|c|}{$\begin{array}{l}\text { All serious infections: J13.9, J18.0, J18.1, J18.9, J22.9, A40.3, G00.1, M00.1, } \\
\mathrm{M} 00.2 \mathrm{~B} / 2 \mathrm{C} / 2 \mathrm{D} / 2 \mathrm{~F} / 2 \mathrm{G} / 2 \mathrm{H} / 2 \mathrm{X}, \mathrm{B} 95.3\end{array}$} \\
\hline \multicolumn{6}{|c|}{$\begin{array}{l}\text { Number of events, relative risk (RR) and ratio of relative risk (RRR) calculated with Generalized } \\
\text { estimated equation (GEE), relative risk reduction (1-RRR), absolute risk reduction (ARR), number } \\
\text { needed to treat (NNT). Pneumonias and all serious pneumococcal infections are shown separately. * } \\
\text { statistical significance }\end{array}$} \\
\hline
\end{tabular}

In the patient group (RA+SpA), the relative risk of pneumonia as well as serious putative pneumococcal infection was significantly reduced, $53 \%$ and $46 \%$ respectively. Among the vaccinated RA patients there was a significant relative risk reduction of pneumonia of $46 \%$. The relative risk reduction of pneumonia among vaccinated SpA patients was 70\%, however not statistically significant. The SpA patients had an absolute risk reduction of both endpoints; $2 \%$ vs $3 \%$, and NNT of 50 and 34 respectively, see Table 2 .

Time to first event before vaccination was significantly shorter among the vaccinated patients for both pneumonia $(p=0.004)$, and for all serious pneumococcal infections $(p=0.05)$. Aftervaccination there was no longer a statistically significant difference in time to first event for either pneumonia $(p=0.12)$ nor for all serious pneumococcal infections $(p=0.09)$ including adjustment for number of the same type of event before vaccination. (Figure 1.A-D).

During the time of follow-up after vaccination, 140 vaccinated patients (24\%) and 100 reference subjects $(4 \%)$ deceased. Time to death was significantly shorter among the vaccinated cohort $(p<0.0001) .15$ patients and 10 reference individuals deceased within 90 days after a registered serious infection, whereof 8 patients and 8 references within 30 days.

Table 3 summarizes results of uni- and multivariate Cox regression models with regard to the time after vaccination. In the univariate predictor analysis several significant risk factors of contracting a serious pneumococcal infection despite vaccination was seen; higher age, RA diagnosis, longer disease duration, higher disease activity at vaccination (DAS28CRP), and treatment with rituximab or prednisolone at vaccination. RA patients were on average 10 years older than the SpA patients and were to a larger extent treated with prednisolone. Gender, smoking at vaccination, treatment with methotrexate or bDMARD other than rituximab was not associated with higher risk of infection after vaccination. 
Table 3

Predictors of all serious infections after vaccination using Cox regression analysis.

\begin{tabular}{|c|c|c|c|}
\hline & P-value & Hazard ratio & $95 \% \mathrm{Cl}$ \\
\hline \multicolumn{4}{|l|}{ Univariate analysis } \\
\hline Age (per year) & $<0.001$ & 1.06 & $1.05-1.07$ \\
\hline Gender (female/male) & 0.100 & 1.23 & $0.96-1.58$ \\
\hline Diagnosis (RA/SpA) & $<0.001$ & 2.46 & $1.89-3.20$ \\
\hline Smoking at vaccination (yes/no) & 0.025 & 1.85 & $1.08-3.16$ \\
\hline Disease duration at vaccination & 0.019 & 1.02 & $1.00-1.04$ \\
\hline DAS28CRP at vaccination & 0.016 & 1.34 & $1.06-1.70$ \\
\hline Methotrexate at vaccination yes/no & 0.785 & 0.94 & $0.58-1.50$ \\
\hline Rituximab at vaccination (yes/no) & 0.096 & 1.82 & $0.90-3.68$ \\
\hline Rituximab and MTX at vacc (yes/no) & 0.945 & 1.04 & $0.33-3.32$ \\
\hline bDMARD at vaccination (yes/no) & 0.652 & 1.13 & $0.67-1.92$ \\
\hline Prednisolone at vaccination (yes/no) & $<0.001$ & 2.63 & $1.64-4.22$ \\
\hline Prednisolone dose (mg/week) & $<0.001$ & 1.02 & $1.01-1.03$ \\
\hline \multicolumn{4}{|l|}{ Multivariate analysis } \\
\hline Age (per year) & $<0.001$ & 1.05 & $1.03-1.08$ \\
\hline Diagnosis (RA/SpA) & 0.008 & 1.48 & $1.11-1.96$ \\
\hline Disease duration at vaccination & 0.670 & 1.00 & $0.99-1.02$ \\
\hline DAS28CRP at vaccination & 0.242 & 1.17 & $0.90-1.52$ \\
\hline Rituximab at vaccination (yes/no) & 0.649 & 1.18 & $0.58-2.42$ \\
\hline Prednisolone at vaccination (yes/no) & 0.004 & 2.04 & $1.26-3.32$ \\
\hline Prednisolone dose (mg/week) & $<0.001$ & 1.02 & $1.01-1.03$ \\
\hline \multicolumn{4}{|c|}{$\begin{array}{l}\text { DAS28CRP, disease activity score using } 28 \text { joint counts, CRP and global health. \# Diagnosis } \\
\text { (RA/SpA), Disease duration, DAS28CRP, Rituximab, Methotrexate, Prednisolone (yes/no) and } \\
\text { Prednisolone dose are analyzed in different regression models adjusted for age. }\end{array}$} \\
\hline
\end{tabular}

When multivariate analysis was applied, only age, RA diagnosis, use of prednisolone and higher prednisolone dose at vaccination remained significant risk factors.

\section{Discussion}


In this report we investigate the effectiveness of one dose of PCV7 among patients with inflammatory arthritis and ongoing anti-rheumatic treatments by assessing the number of registered putative pneumococcal infections up to ten years before and after vaccination, comparing event rates with nonvaccinated reference subjects. The main results were relative risk reductions of pneumonia as well as all serious pneumococcal infections of $53 \%$ and $46 \%$, respectively, among all vaccinated arthritis patients.

The RA patients, who were 10 years older than the SpA patients and affected by the majority of events, showed a relative risk reduction of the two endpoints of $46 \%$ and $37 \%$ respectively. The relative risk reduction of pneumonia among SpA patients was $70 \%$, however not statistically significant, probably due to a low number of events. The incidence of events among the vaccinated SpA patients was higher than their references before vaccination, but lower after vaccination resulting in the absolute risk reduction of all serious pneumococcal infections of $3 \%$, and NNT of 34 . Time to first event before vaccination was significantly shorter among the patients, however no significant difference was seen after vaccination. Treatment with rituximab and higher disease activity at vaccination were associated with contracting infection after vaccination, but after adjustment for age, these factors were no longer significant predictors, possibly due to lack of power. As expected, higher age predicted a significant increase in risk of infection, as well as the diagnosis RA, use and dose of Prednisolone, adjusted for age.

Several studies have evaluated the antibody levels before and shortly after different kinds of pneumococcal immunizations among arthritis patients, concluding that most bDMARDs including antiTNF does not significantly hamper the antibody reaction, whereas MTX and RTX usually does. $(7,9)$ Previous studies have indicated preserved antibody levels following PPV up to 5-10 years, resulting in the recommendation of the polysaccharide revaccination strategy after about 5 years. (10-11) Broyde et al suggested that antibody levels are preserved up to ten years following PPV23 among RA, SpA and AS patients on bDMARD and MTX, questioning the need of revaccination. (12) In 2013, Kapetanovic et al reported a decline in serotype specific antibody back to pre-vaccination levels 1.5 years following immunization with PCV7 among arthritis patients on anti-TNF and MTX, (13) but more studies of longterm preservation of antibody levels following PCV are needed. Our clinical results indicating a long-term protective effect, suggest preserved antibody levels for several years after PCV.

PCV7 includes only seven of the thirty most pathogenic pneumococcal serotypes; $4,6 \mathrm{~B}, 9 \mathrm{~V}, 14,18 \mathrm{C}, 19 \mathrm{~F}$, and 23F. After the introduction of PCV7, several versions of the vaccine with a larger number of serotypes included have been developed: PCV 10,-13, and -15. Studies indicate however cross-reactivity after both PPV and PCV between vaccine serotypes and nonvaccine serotypes. (14-17) Although this phenomenon seems to decrease with age, (18) it could be suggested to be part of the protective effect of immunization seen in our study. While the live attenuated tuberculosis vaccine (BCG) has been shown to induce a nonspecific cross-protection against other pathogens through so called trained innate immunity (19), this has not been shown in pneumococcal vaccines to this date. One could however argue that preventing one infection regardless of the pathogen, reduces the risk of contracting another. Even though this study was performed before the covid -19 pandemic, it could also be worth mentioning the significant inverse 
correlation shown between rates of pneumococcal vaccination and rates of SARS-CoV-2 infections and death. (20)

Our study is not without important limitations. The calculations of event rates based on ICD-codes from a diagnostic register have weaknesses. Apart from pneumococcal infections, the registered events could obviously be representing illness caused by alternative pathogens. However, this possibility of misclassification is estimated to be alike in both patients and reference subjects.

Before inclusion started in 2008, 6 different treatment groups were predefined in order to ensure the inclusion of arthritis patients with a variety of ongoing treatment (see methods). Previous studies have shown reduced antibody response among patients with ongoing methotrexate and rituximab (21), therefore we wanted to include patients with an expected benefit of an immunization. All patients meeting the inclusion criteria and concurrently fitting in to one of the treatment groups were consecutively offered to enter, when arriving for their regular follow-up at the Department of Rheumatology in Lund. However, RA patients in remission without DMARD, on only salazopyrine, hydroxychloroquine or glucocorticoids were not offered to be included, nor were patients not showing up to their doctor's appointments due to low or absent disease activity. $75 \%$ of the included patients were on treatment with bDMARD at the time of vaccination, in comparison to on average $25 \%$ in Swedish RA patients at the time. (22-23) The reference subjects however, were included strictly on the matching criteria (age, sex, arthritis diagnosis) and were to be registered as living in Skåne county 3 years before and 3 years after index date, for the purpose of obtaining their events in the Skåne health care register, implying residual confounding (confounding by indication).

The study protocol did not enable review of the medical records of the reference subjects, limiting the comparisons possible on a treatment group level between vaccinated and non-vaccinated. The number of reference subjects receiving a polysaccharide pneumococcal vaccine (PPV) or a conjugated pneumococcal vaccine (PCV) of any kind during the course of the study is unknown. In 2011 the EULAR recommendations included pneumococcal vaccine in the guidelines (24) but based on previous assessments of pneumococcal vaccine coverage during this time and later, (25-27) and the results presented in this study, we would argue that it probably was a minor proportion.

Overall, we interpret the differences between the groups regarding burden of infectious disease before vaccination date and mortality rate after vaccination date despite the matching on arthritis diagnosis, age, sex and geographical area as a consequence of confounding by indication, including the reference subjects having to be alive at least 3 years after index date (immortal time bias).

Before vaccination, the incidences of events were higher, and the time to first event was shorter among the vaccinated patients compared to the references. The time to first event after vaccination did not differ between the groups on a statistically significant level but the estimates still suggested a tendency of 8-9 months shorter time for the vaccinated. The mortality rates were higher and time to death was shorter in the vaccinated group. Specific information of causes of death was not available to our group, but only a minor number of patients $(n=8)$ and references $(n=8)$ deceased within 30 days following a registered 
serious infection. The calculations are adjusted for a shorter time of follow-up among the vaccinated patients, due to this difference in mortality. Despite the mismatch however, the relative risk reduction of infection was significant among the vaccinated patients. The differences at baseline are clearly a weakness of the study but could be argued to strengthen the result and could possibly underestimate the vaccine efficacy.

In summary, our study showed significant relative risk reductions of both endpoints; pneumonia and all serious putative pneumococcal infections up to ten years after immunization in RA and SpA patients on different antirheumatic treatments despite a higher burden of disease at baseline among the vaccinated patients compared to non-vaccinated reference subjects with arthritis. Higher age, RA diagnosis, and use as well as higher dose of prednisolone were associated with increased risk of infection after vaccination, whereas treatment with methotrexate, rituximab or bDMARD were not.

\section{Conclusions}

One single dose of heptavalent pneumococcal conjugate vaccine may decrease the risk of putative serious pneumococcal infection up to 10 years after vaccination in adult patients with inflammatory arthritis receiving immunomodulating treatment. However, the vaccine does not seem to substantially prolong the time to first serious infection. High age, RA diagnosis, use and higher dose of prednisolone significantly increase the risk of serious pneumococcal infection.

\section{Abbrevations}

$\mathrm{RA}$ : rheumatoid arthritis

SpA : spondylarthropathy

PsA : psoriatic arthritis

DMARDs : disease modifying antirheumatic drugs

bDMARDs biologic disease modifying antirheumatic drugs

NSAID : nonsteroid anti-inflammatory drug

MTX : methotrexate

RTX : rituximab

IRD : inflammatory rheumatic disease

PPV : polysaccharide pneumococcal vaccine

PCV : pneumococcal conjugated vaccine 
$\mathrm{RR}$ : relative risk

RRR : ratio of relative risk

1-RRR : relative risk reduction

ARR : absolute risk reduction

NNT : number needed to treat

GEE : generalized estimated equation

DAS28 : disease activity score using 28 joint counts

CRP : C-reactive protein

$H A Q$ : health assessment questionnaire

$\mathrm{RF}$ : rheumatoid factor

Anti-CCP : anti-cyclic citrullinated peptide

HLA : human leucocyte antigen

ICD : international classification of disease

EULAR : European alliance of associations for rheumatology

BCG : Bacillus Calmette-Guérin

MBC : memory B-cells

\section{Declarations}

\section{Ethical Approval and Consent to participate}

Ethical approval was obtained from the Regional Ethical Review Board at Lund University, Lund, Sweden. (LU 513-01) Oral and written consent of participation was obtained from all patients receiving vaccination before inclusion in the study.

\section{Consent for publication}

Oral and written consent for publication was obtained from all patients receiving vaccination before inclusion in the study. 


\section{Data availability statement}

For reasons related to the legal framework governing the raw data used for this study, individual-level data cannot be freely shared. For reasonable requests for study data, please contact the corresponding author.

\section{Disclosure statement}

Prevenar7 vaccine was provided by Wyeth Pharmaceuticals. The sponsors had no role in study design, data collection, data interpretation or writing of the report. The authors declare no conflicts of interest.

\section{Funding}

The study was supported by grants from the Swedish Rheumatism Association, the Swedish Research Council, the Medical Faculty of the University of Lund, Alfred Österlund's Foundation, The Anna Greta Crafoord Foundation, Greta and Johan Kock's Foundation, the King Gustaf V's 80-year foundation and Skåne University Hospital.

\section{Author contributions}

Conceptualization: MCK, ME, TS, GJ. Data curation and analysis: JN, MCK, J-ÅN, CM, ME. Project administration: MCK, JN. Supervision and validation: all. Writing original draft: JN. Review and editing: all.

\section{Acknowledgements}

Associate professor Pierre Geborek is a former colleague who initiated and contributed to the vaccination projects at the Department of Rheumatology in Lund including the study presented in this paper.

\section{Author's information}

Lund University, Skåne University Hospital, Department of Clinical Sciences Lund, Rheumatology, Lund, Sweden: Johanna Nagel, Jan-Åke Nilsson, Tore Saxne, Meliha C Kapetanovic

Lund University, Skåne University Hospital, Department of Clinical Sciences Lund, Infection Medicine, Lund, Sweden: Göran Jönsson

Lund University, Skåne University Hospital, Faculty of Medicine, Department of Clinical Sciences Lund, Orthopaedics, Clinical Epidemiology Unit, Lund, Sweden : Martin Englund 
Centre of Registers South, Skåne University Hospital, Lund, Sweden : Chanchai Manuswin

\section{References}

1. Blanchard-Rohner G, Pollard A J. Long-term protection after immunization with proteinpolysaccharide conjugate vaccines in infancy. Expert Rev Vaccines 2011 May;10(5):673-84.

2. Friedman M A, Whinthrop K L. Vaccines and Disease-Modifying Antirheumatic Drugs Practical Implications for the Rheumatologist. Rheum Dis Clin N Am 2017 Feb;43(1):1-13.

3. Papadatou I, Spoulou V. Pneumococcal Vaccination in High-Risk Individuals: Are We Doing It Right? Clin Vaccine Immunol. 2016 May; 23(5): 388-395.

4. Furer V, Rondaan C, Heijstek MW, Agmon-Levin N, van Assen S, Bijl M, Breedveld FC, D'Amelio R, Dougados M, Kapetanovic MC, van Laar JM, de Thurah A, Landewé RB, Molto A, Müller-Ladner U, Schreiber K, Smolar L, Walker J, Warnatz K, Wulffraat NM, Elkayam O. 2019 update of EULAR recommendations for vaccination in adult patients with autoimmune inflammatory rheumatic diseases. Annals of the Rheumatic Diseases. 2020 Jan; 79(1):39-52

5. Subesinghe S, Bechman K, Rutherford A I, Goldblatt D, Galloway J. A Systematic Review and Metaanalysis of Antirheumatic Drugs and Vaccine Immunogenicity in Rheumatoid Arthritis. The Journal of Rheumatology 2018 Jun; 45 (6) 733-744.

6. Aliberti S, Mantero M, Mirsaeidi M, Blasi F. The role of vaccination in preventing pneumococcal disease in adults. Clin Microbiol Infect. 2014 May;20 Suppl 5(0 5):52-8

7. Rondaan C, Furer V, Heijstek MW, Agmon-Levin N, Bijl M, Breedveld FC, D'Amelio R, Dougados M, Kapetanovic MC, van Laar JM, Ladefoged de Thurah A, Landewé R, Molto A, Müller-Ladner U, Schreiber K, Smolar L, Walker J, Warnatz K, Wulffraat NM, van Assen S, Elkayam O. Efficacy, immunogenicity and safety of vaccination in adult patients with autoimmune inflammatory rheumatic diseases: a systematic literature review for the 2019 update of EULAR recommendations. RMD Open. 2019 Sep 9;5(2):e001035

8. Jackson LA, Gurtman A, van Cleeff M, Jansen KU, Jayawardene D, Devlin C, Scott DA, Emini EA, Gruber WC, Schmoele-Thoma B. Immunogenicity and safety of a 13-valent pneumococcal conjugate vaccine compared to a 23-valent pneumococcal polysaccharide vaccine in pneumococcal vaccinenaive adults. Vaccine. 2013 Aug 2;31(35):3577-84

9. Bingham CO 3rd, Looney RJ, Deodhar A, Halsey N, Greenwald M, Codding C, Trzaskoma B, Martin F, Agarwal S, Kelman A. Immunization responses in rheumatoid arthritis patients treated with rituximab: results from a controlled clinical trial. Arthritis Rheum. 2010 Jan;62 (1):64-74

10. Mufson M A, Krause HE, Schiffman G. Long-term persistence of antibody following immunization with pneumococcal polysaccharide vaccine. Proc Soc Exp Biol Med. 1983 Jun;173(2):270-5.

11. Rasmussen S L, Fuursted K, Nielsen K A, Laurberg N P, Sørensen M B, Fagerberg S K, Leutscher $P$, Rasmussen C. Pneumococcal antibody protection in patients with autoimmune inflammatory rheumatic diseases with varying vaccination status. Scand J Rheumatol. 2020 Sep;49:5, 353-360. 
12. Broyde A, Arad U, Madar-Balakirski N, Paran D, Kaufman I, Levartovsky D, Wigler I, Caspi D, Elkayam O. Longterm efficacy of an antipneumococcal polysaccharide vaccine among patients with autoimmune inflammatory rheumatic diseases. J Rheumatol . 2016 Feb;43:267-72.

13. Kapetanovic M C, Saxne T, Truedsson L, Geborek P. Persistence of antibody response 1.5 years after vaccination using 7-valent pneumococcal conjugate vaccine in patients with arthritis treated with different antirheumatic drugs. Arthritis Res Ther. 2013 Jan 4;15(1):R1.

14. Cooper D, Yu X, Sidhu M, Nahm MH, Fernsten P, Jansen KU. The 13-valent pneumococcal conjugate vaccine (PCV13) elicits cross-functional opsonophagocytic killing responses in humans to Streptococcus pneumoniae serotypes 6C and 7A. Vaccine. 2011 Sep 22;29(41):7207-11.

15. Cho H K, Park I H, Burton R L, Kim K H. Impact of IgM Antibodies on Cross-Protection against Pneumococcal Serogroups 6 and 19 after Immunization with 7-Valent Pneumococcal Conjugate Vaccine in Children. J Korean Med Sci. 2016 Jun;31(6):950-6.

16. Hausdorff W P, Hoet B, Schuerman L. Do pneumococcal conjugate vaccines provide any crossprotection against serotype 19A? BMC Pediatr. 2010 Feb 2; 10:4.

17. Lee H, Cha J H, Nahm M H, Burton R L, Kim K-H. The 7-valent pneumococcal conjugate vaccine elicits cross-functional opsonophagocytic killing responses to Streptococcus pneumoniae serotype 6D in children. BMC Infect Dis. 2013 Oct 10;13:474.

18. Kim H W, Lee S, Kim K-H. Serotype 6B from a pneumococcal polysaccharide vaccine induces crossfunctional antibody responses in adults to serotypes 6A, 6C, and 6D. Medicine (Baltimore). 2016 Sep;95(37):e4854

19. Covián C, Fernández-Fierro A, Retamal-Díaz A, Díaz FE, Vasquez AE, Lay MK, Riedel CA, González PA, Bueno SM, Kalergis AM. BCG-Induced Cross-Protection and Development of Trained Immunity: Implication for Vaccine Design. Front Immunol. 2019 Nov 29;10:2806.

20. Root-Bernstein R. Possible Cross-Reactivity between SARS-CoV-2 Proteins, CRM197 and Proteins in Pneumococcal Vaccines May Protect Against Symptomatic SARS-CoV-2 Disease and Death. Vaccines (Basel). 2020 Sep 24;8(4):559.

21. Hua C, Barnetche T, Combe B, Morel J. Effect of Methotrexate, Anti-Tumor Necrosis Factor a, and Rituximab on the Immune Response to Influenza and Pneumococcal Vaccines in Patients With Rheumatoid Arthritis: A Systematic Review and Meta-Analysis. Arthritis Care Res (Hoboken). 2014 Jul;66(7):1016-26.

22. Neovius M, Simard JF, Askling J; ARTIS study group. Nationwide prevalence of rheumatoid arthritis and penetration of disease-modifying drugs in Sweden. Ann Rheum Dis. 2011 Apr;70(4):624-9.

23. Neovius M, Sundström A, Simard J, Wettermark B, Cars T, Feltelius N, Askling J, Klareskog L; ARTIS Study Group. Small-area variations in sales of TNF inhibitors in Sweden between 2000 and 2009. Scand J Rheumatol. 2011 Jan;40(1):8-15

24. van Assen S, Agmon-Levin N, Elkayam O, Cervera R, Doran MF, Dougados M, Emery P, Geborek P, Ioannidis JP, Jayne DR, Kallenberg CG, Müller-Ladner U, Shoenfeld Y, Stojanovich L, Valesini G, 
Wulffraat NM, Bijl M. EULAR recommendations for vaccination in adult patients with autoimmune inflammatory rheumatic diseases. Ann Rheum Dis. 2011 Mar;70(3):414-22.

25. Hmamouchi I, Winthrop K, Launay O, Dougados M. Low rate of influenza and pneumococcal vaccine coverage in rheumatoid arthritis: data from the international COMORA cohort. Vaccine. 2015 Mar 17;33(12):1446-52.

26. Nguyen $\mathrm{M}$, Lindegaard $\mathrm{H}$, Hendricks $\mathrm{O}$, Friis-Møller $\mathrm{N}$. Factors associated with influenza and pneumococcal vaccine uptake among rheumatoid arthritis patients in Denmark invited to participate in a pneumococcal vaccine trial (Immunovax_RA). Scand J Rheumatol. 2017 Nov;46(6):446-453.

27. Qendro T, de la Torre ML, Panopalis P, Hazel E, Ward BJ, Colmegna I, Hudson M. Suboptimal Immunization Coverage among Canadian Rheumatology Patients in Routine Clinical Care. $J$ Rheumatol. 2020 May 1;47(5):770-778.

\section{Figures}
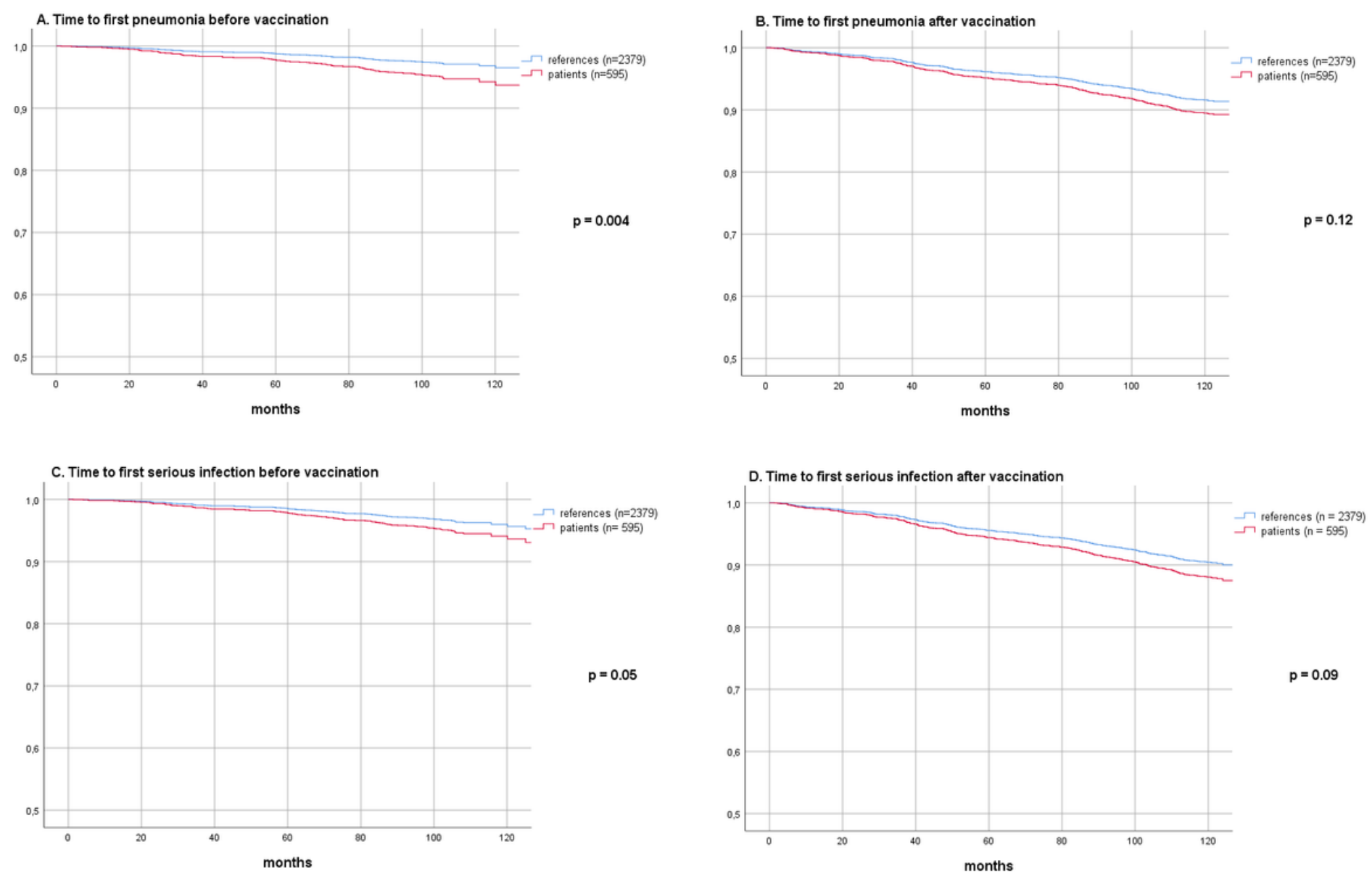

\section{Figure 1}

Legend not included with this version 